\title{
Somnologie
}

\section{Bei Schlafstörungen leidet auch die Arbeitsfähigkeit}

Klösch G et al. Schlaf und Arbeitsleben. Ergebnisse einer Studie der Bundesarbeits-

kammer Österreich (BAK-Studie). Somnologie 2010;14:32-40

\section{Hintergrund und Fragestellung}

Ein nicht erholsamer Schlaf beeinträchtigt die Arbeitsleistung und die Stressbewältigung. Ein gestörter Schlaf gilt auch als einer der Risikofaktoren für ein Burnout-Syndrom. Eine weitere Folge kann eine gesteigerte Tagesmüdigkeit sein, die neben einer verminderten Aufmerksamkeits- und Konzentrationsleistung auch als eine Ursache für Arbeits- und Verkehrsunfälle angesehen wird [1]. Die vorliegende Studie untersuchte die Auswirkungen eines gestörten Schlafs auf die Arbeitszufriedenheit, die Arbeitsfähigkeit und die allgemeine Gesundheit.

\section{Methodik}

Der Studie lagen die Ergebnisse einer Umfrage der Bundesarbeitskammer Österreich an 4214 Arbeitnehmern in Hinblick auf Ein- und Durchschlafstörungen und arbeitsbedingte Müdigkeit zugrunde. 52,5\% der Befragten waren männlich, 15,8\% waren jünger als 40 Jahre und 1,7\% mindestens 60 Jahre alt. Die Befragten arbeiteten im Gesundheitswesen, im Tourismus, in der Bau- und Metallindustrie oder im Handels- und Bankwesen.

\section{Ergebnisse}

Rund 23\% der Befragten gaben an, mehrmals pro Woche an Ein- und Durchschlafstörungen zu leiden. Frauen über 40 Jahren waren häufiger betroffen. Außerdem nahm die Häufigkeit von Schlafstörungen mit dem Alter zu. So hatten 34\% der Befragten zwischen 55 und 59 Jahren Schlafstörungen.

Qualifizierte und leitende Angestellte litten am wenigsten unter Schlafstörungen $(<20 \%)$. Arbeitnehmer mit Schlafstörungen berichteten signifikant häufiger, unter einer ermüdenden Arbeit zu leiden (44,7 vs. 19,4\%).

Schlafstörungen hatten signifikant negative Auswirkungen auf den allgemeinen
Gesundheitszustand, die Arbeitsfähigkeit und die arbeitsbedingte Müdigkeit. Das Alter und das Geschlecht spielten dabei eine wesentliche Rolle, nicht jedoch der Beruf, die Branche, der familiäre Status und die Arbeitszufriedenheit.

\section{Schlussfolgerung}

Im öffentlichen Diskurs muss den Konsequenzen von nicht erholsamem Schlaf für die Arbeitswelt deutlich mehr Aufmerksamkeit geschenkt werden.

\section{Kommentar}

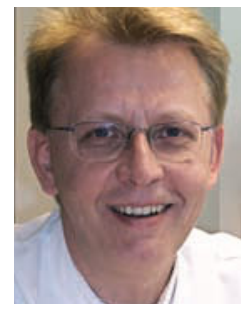

Prof. Dr. med. Kurt Rasche, Wuppertal

Diese Studie befasste sich mit einem volkswirtschaftlich und gesundheitspolitisch wichtigen Thema. Ein nicht erholsamer Schlaf hat einen bedeutenden Einfluss auf die Leistungsfähigkeit des Einzelnen. Da Schlafstörungen mit dem Alter an Häufigkeit zunehmen, kommt es zu einem additiven, wenn nicht sogar potenzierenden Effekt von Auswirkungen der biologischen Alterung und der zunehmenden Schlafstörung. Diesem Umstand wird in der Arbeitswelt sicherlich noch zu wenig Rechnung getragen, da im Allgemeinen die Arbeitszeiten relativ rigide unabhängig vom Alter festgelegt sind. Möglicherweise müssten daher von arbeitsmedizinischer Seite viel früher müdigkeitsbedingte Defizite der Arbeitnehmer erkannt, die Ursachen erfasst und, wenn möglich, betriebsorganisatorische Konsequenzen gezogen werden.

\section{Literatur}

1. Akerstedt T et al. J Psychosom Res. 2004;54:427-433 